\title{
Association of estrogen receptor alpha gene polymorphisms and lifestyle factors with calcaneal quantitative ultrasound and osteoporosis in postmenopausal Vietnamese women
}

\author{
Tran Quang Binh · Toshikatsu Shinka $\cdot$ Nguyen Cong Khan · \\ Vu Thi Thu Hien · Nguyen Thi Lam · Le Bach Mai · Takuro Nakano • \\ Masako Sei $\cdot$ Shigeru Yamamoto $\cdot$ Masayo Nakamori $\cdot$ Yutaka Nakahori
}

Received: 5 June 2006/ Accepted: 17 August 2006/ Published online: 14 September 2006

(C) The Japan Society of Human Genetics and Springer 2006

\begin{abstract}
Abtract Genetic and lifestyle factors are important in the pathogenesis of osteoporosis. We investigated the relationships of $P v u \mathrm{II}$ and $X b a \mathrm{I}$ polymorphisms of the estrogen receptor alpha $(\mathrm{ER}-\alpha)$ gene, lifestyle factors with speed of sound at the calcaneus (calcaneal SOS) and osteoporosis in a population-based study of 140 healthy postmenopausal women. By an analysis of covariates, women with higher copies of $\mathrm{P}$ or $\mathrm{X}$ alleles had higher calcaneal SOS compared with others $(P=0.012$, PP vs. pp; $P=0.045, \mathrm{Xx}$ vs. $\mathrm{xx})$. Women with lower copies of px or higher copies of PX haplotypes had higher calcaneal SOS compared with others $(P=0.021,0$ px vs. $2 \mathrm{px} ; P=0.011,1$ PX vs. 0 PX). The px and PX haplotypes, age and years since menopause were found to be independent predictors of calcaneal SOS in multiple linear regression models. Using logistic regression, we found an increased osteoporosis
\end{abstract}

T. Q. Binh · T. Shinka · T. Nakano · M. Sei ·

Y. Nakahori $(\bowtie)$

Department of Human Genetics and Public Health,

Faculty of Medicine, Graduate School of Proteomics,

The University of Tokushima, 3-18-15 Kuramoto-cho,

Tokushima 770-8503, Japan

e-mail: nakahori@basic.med.tokushima-u.ac.jp

T. Q. Binh

National Institute of Hygiene and Epidemiology,

Hanoi, Vietnam

N. C. Khan · V. T. T. Hien · N. T. Lam - L. B. Mai

National Institute of Nutrition, Hanoi, Vietnam

S. Yamamoto · M. Nakamori

Department of International Public Health Nutrition, Institute of Health Biosciences, The University of

Tokushima, Tokushima, Japan risk with evidence for a px haplotype dose effect $(\mathrm{OR}=2.82,95 \% \mathrm{CI}=1.50-5.31, P=0.001)$ and for a $\mathrm{PX}$ haplotype dose effect $(\mathrm{OR}=0.42,95 \% \mathrm{CI}=0.19-0.93$, $P=0.033)$. An increased educational level was associated with a reduced risk of osteoporosis $(P=0.035$ in the model with px, $P=0.044$ in the model with PX). In conclusion, the present study suggests that $P v u \mathrm{II}$ and $X b a$ I polymorphims of the ER- $\alpha$ gene, age, years since menopause and educational level are associated with bone density, as assessed by calcaneal SOS, and osteoporosis in postmenopausal Vietnamese women.

Keywords Estrogen receptor- $\alpha$ gene $\cdot$ Lifestyle factors · Osteoporosis - Quantitative ultrasound . Postmenopausal Vietnamese women

\section{Introduction}

Osteoporosis is a major health problem worldwide, particularly in the elderly. More than $40 \%$ of postmenopausal women, on average, will suffer at least one osteoporotic fracture (Spencer et al. 1986). It is also a common complex disorder that involves environmental and genetic factors and results in an increased risk of fracture. The environmental risk factors of osteoporosis have been reported in previous studies as low calcium intake, vitamin D deficiency, excessive alcohol, smoking and lack of physical activity (Anthony and Kristina 2003). Several studies have proved the dominant role of the genetic factors in the pathogenesis of osteoporosis. Studies in twins and parent-offspring have shown that additive genetic effects account for $60-80 \%$ of the population variance and stature (Giguere and Rousseau 2000; Nordström and Lorentzon 
1999). Genetic linkage studies (Johnson et al. 1997; Devoto et al. 1998) and candidate-gene association studies have identified loci and candidate genes that are involved in the regulation of bone mass and the pathogenesis of osteoporotic fractures (Morrison et al. 1994; Kobayashi et al. 1996; Masi et al. 1998; Gennari et al. 2005). One such candidate gene is the estrogen receptor alpha $(E R-\alpha)$. The importance of ER- $\alpha$ in the regulation of bone mass has been indicated by the occurrence of osteoporosis in a man with mutations in the coding region of ER- $\alpha$ (Smith et al. 1994) and by the observation that the bone mineral density (BMD) of mice lacking a functional ER- $\alpha$ gene is $20-25 \%$ less than that of wild-type mice (Korach 1994). Several genetic variations in the ER- $\alpha$ gene have been described, and relationships of these polymorphisms with BMD and quantitative ultrasound (QUS) parameters have been reported, but the results have been inconsistent, pointing to the possibility that gene-gene and gene-environment interactions may present important confounding factors (Kobayashi et al. 1996; Han et al. 1997; Patel et al. 2000; Kim et al. 2001; Van Meurs et al. 2003; Koh et al. 2004; Albagha et al. 2005; Mitra et al. 2006). Moreover, there are few studies that have analyzed the relationship of both ER- $\alpha$ polymorphisms and environmental factors to osteoporosis. Here, we take into account anthropometric measurements and life style factors (such as weight, height, age, years since menopause, educational level, occupation, smoking and drinking alcohol) in analyzing the association of ER- $\alpha$ with osteoporosis.

To assess bone status, QUS of the bone has been proposed as an alternative method for noninvasive assessment of skeletal status, as it reflects not only the bone mass, but also the qualitative aspects of bone tissue such as elasticity, structure and geometry (Gluer 1997). The heritability estimates of the QUS parameters are also comparable with those of BMD, ranging between 53 and $74 \%$ at the calcaneus (Danielson et al. 1999; Howard et al. 1998). More importantly, the proportion of postmenopausal women classified as normal, osteopenic or osteoporotic was similar when using either BMD or QUS measurements (Frost et al. 2001; Huopio et al. 2004). However, in contrast to the many reports using BMD, genetic studies using QUS measurements are extremely rare. In addition, to the best of our knowledge, there is still a dearth of data on the genetic factors of osteoporosis in Vietnam. Therefore, we performed a population-based study to investigate the association of the $P v u \mathrm{II}$ and $X b a \mathrm{I}$ genotype, haplotype of ER- $\alpha$ gene as well as environmental factors with osteoporosis and bone density assessed by SOS at the calcaneus in postmenopausal Vietnamese women.

\section{Subjects and methods}

\section{Subjects}

First, 500 postmenopausal women living in a rural area of the Hai Duong province were screened from two communes by questionnaire. Women who had one or more of the following factors were excluded from the study: (1) current cancer or hyperparathyroidism; (2) a kidney stone in the past 5 years, renal disease, or bilateral hip surgery; (3) therapy with a bisphosphonate, calcitonin, estrogen, tamoxifen, or testosterone in the past 6 months or fluoride in the past 2 years; (4) laboratory evidence of kidney or liver disease. As a result, 140 healthy postmenopausal women who met the criteria of the study were recruited for the study. None of the recruited subjects had ever used any bisphosphonates in their medical history. The Ethics Committee of the National Institute of Nutrition, Vietnam, and the Ethnical Committee of Tokushima University, Japan, approved the study. All participants provided written informed consent before entering the study.

\section{Measurements}

All participants completed a structured questionnaire. Data were collected on the anthropometric measurements, current age, age at menarche, age at menopause, ethnicity, educational level, occupation, medical and reproductive history, and smoking and drinking history. The lifelong occupation was defined as the occupation that the subject engaged in most frequently in her life. It was classified as heavy work (farmers; manual workers), office work (office clerks and other sedentary jobs) or domestic work (housewife). The educational level was categorized into three groups according to the number of years of schooling: low level ( $\leq 5$ years), medium level (6-8 years) and high level ( $\geq 9$ years).

Bone mass was assessed by speed of sound $(\mathrm{m} / \mathrm{s})$ at the calcaneus using a QUS device (CM-100; ELK Corporation, Tokyo, Japan) as described by Hien et al. (2005). The precision error (percent coefficient of variation) using the phantom technique was $0.15 \%$ and, in vivo, was $0.27 \%$ (Hien et al. 2005). The $T$-score for each subject was calculated by using the peak speed-of-sound value for a defined population of young adults, and its standard deviation, with the following equation (Cheng et al. 1999): $T$-score $=($ speed of sound $_{\text {subject }}-$ speed of sound ${ }_{\text {peak }}$ value for young adults $) /$

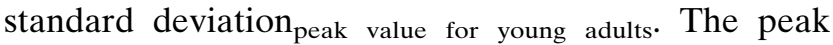
speed-of-sound value for young adults (speed-of-sound peak value for young adults) was found by estimating the 
peak bone mass, which was itself defined as the average maximum bone mass achieved by young, healthy, sex- and race-matched adults (Frost et al. 1999). The speed-of-sound peak value for young adults and standard deviation $_{\text {peak value for young adults }}$ were used from a previous large population-based study (Hien et al. 2005). A person was classified as having osteoporosis if her $T$-score was $\leq-1.8$, and as normal if the score was $>-1.8$.

\section{Genotyping}

Peripheral blood samples were obtained from each woman, and genomic DNA was extracted from peripheral blood leukocytes, using the QIA amp DNA blood kit (Qiagen GmbH, Hilden, Germany). The $P v u I I$ (rs2234693) and $\mathrm{XbaI}$ (rs9340799) polymorphisms are $45 \mathrm{bp}$ apart and located approximately 400 bp upsteam of exon 2 of the ER- $\alpha$ gene. ER- $\alpha$ genotypes and haplotypes were determined with the restriction fragment length polymorphism (RFLP)PCR analysis, as previously described (Van Meurs et al. 2003). The PCR products were digested at $37^{\circ} \mathrm{C}$ for 90 min with 5 units of PvuII and 7 units of $X b a \mathrm{I}$ restriction enzymes (New England Biolabs). Haplotypes for the individuals carrying the PpXx genotype were determined by double digestion with both $P v u \mathrm{II}$ and $X b a \mathrm{I}$ restriction enzymes. The digested products were analyzed by $3 \%$ agarose gel electrophoresis in $0.5 \times \mathrm{TBE}$ buffer at $100 \mathrm{~V}$ for $40 \mathrm{~min}$, and ethidium bromide staining was used. By convention, the small $p$ and $\mathrm{x}$ denote the presence of $P v u \mathrm{II}$ and $X b a \mathrm{I}$ restriction sites, respectively; the capital $\mathrm{P}$ and $\mathrm{X}$ indicate the absence of $P v u \mathrm{II}$ and $X b a \mathrm{I}$ restriction sites, respectively. Four haplotype alleles for $P v u \mathrm{II}$ and $X b a \mathrm{I}$ were encoded as px (1), PX (2), Px (3) and pX (4).

Statistical analysis

We used the general linear models (GLM) for univariate analysis to evaluate the relationships between calcaneal SOS and ER- $\alpha$ genotypes and haplotypes. The raw SOSs were adjusted by regression for covariates of the height, weight, age, years since menopause, and number of children. The educational level was entered in models as a random factor. Multiple group comparisons were made by one-way ANOVA. Groupgroup differences were then assessed by the post hoc test of Bonferroni. Binary logistic regression analysis was used to test several models for the associations of osteoporosis to the $P v u \mathrm{II}$ and $X b a \mathrm{I}$ genotypes, haplotypes of the ER- $\alpha$ gene and other variables. Here, data are presented as the odds ratios with $95 \%$ confidence intervals (CI). Multiple linear regression was used to test for independent predictors for calcaneal SOS, entering the $P v u \mathrm{II}-X b a \mathrm{I}$ haplotypes, age, years since menopause, weight, height and educational level into the model. The independent distribution of errors was assessed by an analysis of residuals. The chi-square $\left(\chi^{2}\right)$ test was used to assess the distribution of $P v u \mathrm{II}, X b a \mathrm{I}$ genotypes and $P v u \mathrm{II}-X b a \mathrm{I}$ haplotypes for HardyWeinberg equilibrium. Associations were considered statistically significant at $P<0.05$ for all the analyses. All statistical procedures were performed using SPSS version 11.0 (SPSS, Inc., Chicago, IL).

\section{Results}

Characteristics of the subjects

Of the 140 subjects in the study, 137 (98\%) were farmers and manual workers, and the others were office clerks. All subjects were healthy women who did not smoke and drink alcohol. The mean $( \pm \mathrm{SD})$ of age, age at menarche and age at menopause of the study group were $55.6 \pm 3.8,16.7 \pm 2.1$ and $47.7 \pm 3.4$ years, respectively. The characteristics of subjects according to the $P v u \mathrm{II}$ and $X b a \mathrm{I}$ genotypes of the ER- $\alpha$ gene are shown in Table 1 . We found no statistically significant differences among the three groups of either polymorphism in the weight, height, BMI, age, age at menopause, years since menopause and number of children.

ER- $\alpha$ genotype and haplotype frequencies

The frequencies of the $P v u \mathrm{II}$ and $X b a \mathrm{I}$ genotypes of the ER- $\alpha$ gene were as follows: PP, $15.7 \%(n=22)$; Pp, $47.1 \%(n=66)$; pp, 37.2\% $(n=52)$; XX, 3.6\% $(n=5) ; \mathrm{Xx}$, $32.1 \%(n=45) ; \mathrm{xx}, 64.3 \%(n=90)$. The frequencies of $P v u \mathrm{II}-\mathrm{Xba \textrm {I }}$ haplotypes were as follows: $\mathrm{px}, 60.7 \%$; PX, $19.6 \%$; Px, 19.6\%; pX, $0 \%$. The frequencies of $P v u \mathrm{II}-$ $\mathrm{XbaI}$ diplotypes were: $37.1,25.7,24.1,3.6,6.4$ and $5.7 \%$ for px-px, px-PX, px-pX, PX-PX, PX-Px and Px-Px, respectively. The distributions of the $P v u \mathrm{II}, X b a \mathrm{I}$ genotypes and the $P v u \mathrm{II}-X b a \mathrm{I}$ haplotype genotypes were compatible with a population in Hardy-Weinberg equilibrium.

Associations of the PvuII and XbaI polymorphisms, PvuII-XbaI haplotype and lifestyle factors with calcaneal SOS

To investigate the relationship between the individual $P v u \mathrm{II}, X b a \mathrm{I}$ polymorphisms and $P v u \mathrm{II}-X b a \mathrm{I}$ haplotype of the ER- $\alpha$ gene with calcaneal SOS, we used the 
Table 1 Characteristics of the subjects according to the $P v u \mathrm{II}$ and $X b a \mathrm{I}$ genotypes of the ER- $\alpha$ gene $(n=140)$

\begin{tabular}{|c|c|c|c|c|c|c|c|c|}
\hline & \multicolumn{3}{|l|}{$P v u \mathrm{II}$} & \multirow[t]{2}{*}{$P$ value } & \multicolumn{3}{|l|}{$X b a \mathrm{I}$} & \multirow[t]{2}{*}{$P$ value } \\
\hline & $\mathrm{PP}(n=22)$ & $\operatorname{Pp}(n=66)$ & pp $(n=52)$ & & $\mathrm{XX}(n=5)$ & $\mathrm{Xx}(n=45)$ & $\mathrm{xx}(n=90)$ & \\
\hline Age (years) & $56.1 \pm 3.4$ & $54.9 \pm 3.6$ & $56.3 \pm 4.1$ & 0.123 & $54.8 \pm 3.7$ & $54.8 \pm 3.8$ & $56.1 \pm 3.8$ & 0.141 \\
\hline Age at menopause (years) & $47.3 \pm 3.1$ & $47.5 \pm 3.8$ & $48.0 \pm 3.1$ & 0.665 & $48.2 \pm 3.3$ & $47.4 \pm 3.6$ & $47.8 \pm 3.4$ & 0.780 \\
\hline Years since menopause & $8.8 \pm 3.2$ & $7.4 \pm 3.1$ & $8.3 \pm 3.6$ & 0.162 & $6.6 \pm 1.1$ & $7.4 \pm 3.2$ & $8.3 \pm 3.5$ & 0.195 \\
\hline Height $(\mathrm{cm})$ & $148.4 \pm 6.0$ & $149.4 \pm 4.7$ & $149.2 \pm 5.1$ & 0.712 & $150.0 \pm 2.7$ & $149.3 \pm 5.5$ & $149.0 \pm 4.9$ & 0.901 \\
\hline Weight (kg) & $46.2 \pm 7.7$ & $46.3 \pm 6.7$ & $44.3 \pm 5.8$ & 0.234 & $50.5 \pm 6.8$ & $45.9 \pm 6.7$ & $45.0 \pm 6.4$ & 0.169 \\
\hline BMI $\left(\mathrm{kg} / \mathrm{m}^{2}\right)$ & $20.9 \pm 2.6$ & $20.7 \pm 2.8$ & $19.9 \pm 2.2$ & 0.134 & $22.5 \pm 3.0$ & $20.6 \pm 2.6$ & $20.2 \pm 2.5$ & 0.151 \\
\hline No. of children & $3.8 \pm 1.7$ & $3.2 \pm 1.4$ & $3.6 \pm 1.5$ & 0.20 & $4.4 \pm 1.1$ & $3.4 \pm 1.3$ & $3.4 \pm 1.6$ & 0.344 \\
\hline
\end{tabular}

Data are presented as the mean \pm SD. The number in parenthesis indicates the number of subjects in each genotype group $P$ values were obtained by the one-way ANOVA test

general linear model (GLM) univariate analysis in which calcaneal SOS was presented as the mean $\pm \mathrm{SE}$ and adjusted for the weight, height, age, years since menopause, number of children, and educational level. As shown in Fig. 1, calcaneal SOS was higher in women with the PP genotype than in those with pp genotypes $(P=0.012$, ANCOVA). On the other hand, calcaneal SOS was lower in women with the $\mathrm{xx}$ genotype than in those with $\mathrm{Xx}$ genotypes $(P=0.045$, ANCOVA). The above analyses resulted in the hypothesis that there was an association between the number copies of PX and/or px haplotypes and calcaneal SOS. In view of this, we next evaluated the relation between each of the three haplotypes (px, PX and Px) and calcaneal SOS (Fig. 2). Calcaneal SOS was significantly lower in the 2-px and 0-PX groups compared to the 0 -px group and 1-PX groups, respectively $(P=0.021,2$ px vs. 0 px; $P=0.011,0$ PX vs. 1 PX, ANCOVA). Carries with higher copy of px tended to have lower calcaneal SOS, while carries with higher copy of PX were prone to have higher calcaneal SOS. A similar analysis showed no association between number copies of the Px haplotype and calcaneal SOS. In addition, the calcaneal SOS was lower in the low educational level compared to the medium level and high level $(P=0.038$ and 0.002, respectively, one-way ANOVA). After adjusting for confounding factors, a significant difference remained between the low educational level and medium educational level $(P=0.004$ ANCOVA, data not shown).

Risk factors associated with osteoporosis and independent predictors for calcaneal SOS

To assess potential risk variables of osteoporosis, we used binary logistic regression in the models, including the copy number of the px or PX haplotype, age, years since menopause, number of children, weight, height and educational level (Table 2). As shown in the model 1 , the copy number of the px haplotype and years since menopause were positively related to the risk of osteoporosis ( $P=0.007,2$ px vs. 0 px; $P=0.045$, respectively). We found an increased osteoporosis risk with evidence for a px haplotype dose effect with an odds ratio of 2.82 (95\% CI=1.50-5.31, $P=0.001$ ). On the other hand, the model 2 showed that the copy number of the PX haplotype was negatively related to the risk of osteoporosis $(\mathrm{OR}=0.42,95 \% \mathrm{CI}=0.19-0.93, P=0.033)$. Increased years since menopause was associated with a significantly increased risk of osteoporosis in model 1 ( $\mathrm{OR}=1.16,95 \% \mathrm{CI}=1.01-1.34, P=0.045)$, but not in the model $2(\mathrm{OR}=1.13,95 \% \mathrm{CI}=0.98-1.30, P=0.087)$. In
Fig. 1 Calcaneal SOS in postmenopausal women according to the $P v u \mathrm{II}$ and $X b a \mathrm{I}$ genotypes of the ER- $\alpha$ gene. SOS values are the means \pm SE and are adjusted for weight, height, age, years since menopause, number of children and educational level. $P$ value obtained by ANCOVA

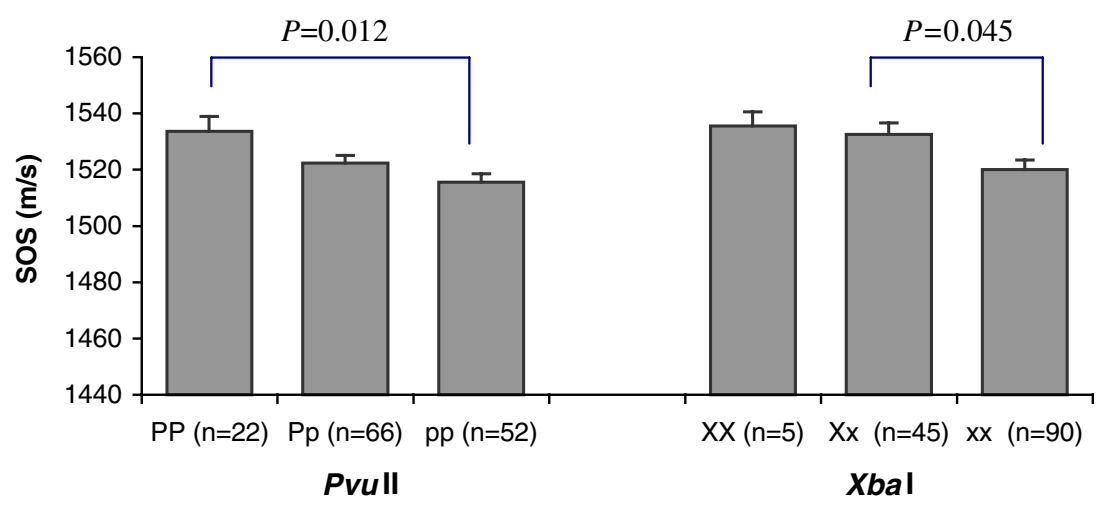


Fig. 2 Calcaneal SOS in postmenopausal women according to $P v u \mathrm{II}-X b a \mathrm{I}$ haplotypes of the ER- $\alpha$ gene. SOS values are the means \pm SE, and are adjusted for weight, height, age, years since menopause, number of children and educational level. $P$ value obtained by ANCOVA

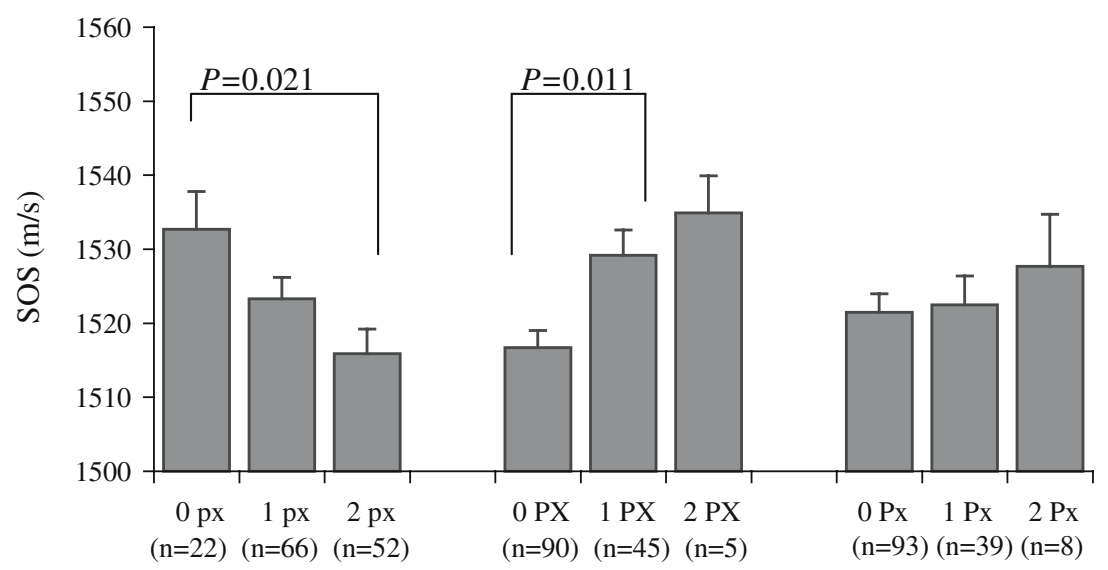

Haplotype

Table 2 Risk factors for osteoporosis assessed by calcaneal SOS in Vietnamese postmenopausal women

\begin{tabular}{|c|c|c|c|c|c|c|c|}
\hline \multicolumn{4}{|l|}{ Model 1} & \multicolumn{4}{|l|}{ Model 2} \\
\hline Factors & Odds ratio & $95 \% \mathrm{CI}^{\mathrm{a}}$ & $P$ value ${ }^{\mathrm{b}}$ & Factors & Odds ratio & $95 \% \mathrm{CI}^{\mathrm{a}}$ & $P$ value $^{\mathrm{b}}$ \\
\hline Number of px & 2.82 & $1.50-5.31$ & 0.001 & Number of PX & 0.42 & $0.19-0.93$ & 0.033 \\
\hline $0 \mathrm{px}$ & 1.00 & - & - & $0 \mathrm{PX}$ & 1.00 & - & - \\
\hline $1 \mathrm{px}$ & 1.51 & $0.42-5.45$ & 0.527 & $1 \mathrm{PX}$ & 0.35 & $0.14-0.88$ & 0.026 \\
\hline $2 \mathrm{px}$ & 6.00 & $1.61-22.1$ & 0.007 & $2 \mathrm{PX}$ & 0.35 & $0.03-3.98$ & 0.401 \\
\hline Years since menopause & 1.16 & $1.01-1.34$ & 0.045 & Years since menopause & 1.13 & $0.98-1.30$ & 0.087 \\
\hline Age & 1.09 & $0.94-1.26$ & 0.258 & Age & 1.09 & $0.95-1.26$ & 0.229 \\
\hline Educational level & 0.54 & $0.30-0.96$ & 0.035 & Educational level & 0.57 & $0.33-0.98$ & 0.044 \\
\hline Low level & 1.00 & - & - & Low level & 1.00 & - & - \\
\hline Medium level & 0.60 & $0.23-1.61$ & 0.315 & Medium level & 0.70 & $0.28-1.79$ & 0.459 \\
\hline High level & 0.27 & $0.08-0.88$ & 0.030 & High level & 0.29 & $0.09-0.93$ & 0.037 \\
\hline Weight & 1.02 & $0.95-1.09$ & 0.683 & Weight & 0.98 & $0.93-1.07$ & 0.951 \\
\hline Height & 1.05 & $0.96-1.16$ & 0.265 & Height & 1.07 & $0.98-1.17$ & 0.152 \\
\hline Number of children & 1.22 & $0.93-1.60$ & 0.152 & Number of children & 1.22 & $0.94-1.58$ & 0.142 \\
\hline
\end{tabular}

a $95 \%$ confidence interval of odds ratio

b $P$ value obtained by logistic regression

both models, an increased educational level was associated with a reduced risk of osteoporosis $(P=0.035$ in model 1 and $P=0.044$ in model 2 ), while there were no associations of the age, weight, height and number of children with a risk of osteoporosis.

To assess independent predictors for calcaneal SOS, we used multiple linear regression, entering the px or PX haplotypes, age, years since menopause, weight, height, number of children and educational level into the models. The models shown in Table 3 explained 23.8 and $25.8 \%$ of the total observed variance in calcaneal SOS according to the copy number of the px and PX haplotypes, respectively. However, the copy number of the px and PX haplotypes, age, and years since menopause were the most significant independent predictors of calcaneal SOS. Further stepwise multiple linear regression analyses showed that the $\mathrm{px}$ and PX haplotypes accounted for approximately 5 and $7 \%$ at the calcaneal SOS, while the age and years since menopause accounted for about 4 and $6 \%$, respectively.

\section{Discussion}

In this study, we found a statistically significant association between calcaneal SOS and the ER- $\alpha P v u I I$ and $X b a \mathrm{I}$ polymorphisms when they were analyzed individually and by the copy number of the px and PX haplotypes in postmenopausal women.

ER- $\alpha$ appears to be the major receptor mediating estrogen action in bone, and it has a prominent effect on the regulation of bone turnover and the maintenance of bone mass (Gennari et al. 2005), but association studies to date have yielded inconsistent results. First, Kobayashi et al. (1996) reported no significant difference in BMD values in Japanese women in relation to individual $P v u I I$ and $X b a I$ polymorphisms. 
Table 3 Multiple linear regression analysis of calcaneal SOS and independent variables

\begin{tabular}{|c|c|c|c|c|c|}
\hline \multicolumn{3}{|l|}{ Model 3} & \multicolumn{3}{|l|}{ Model 4} \\
\hline Predictors & Coefficient & $P$ value & Predictors & Coefficient & $P$ value \\
\hline No. of px haplotype & -9.074 & 0.002 & No of PX haplotype & 13.243 & 0.001 \\
\hline Years since menopause & -1.445 & 0.043 & Years since menopause & -1.155 & 0.102 \\
\hline Age (years) & -1.722 & 0.011 & Age (years) & -1.669 & 0.013 \\
\hline Educational level & 4.629 & 0.092 & Educational level & 4.736 & 0.081 \\
\hline Weight $(\mathrm{kg})$ & 0.140 & 0.693 & Weight $(\mathrm{kg})$ & 0.152 & 0.662 \\
\hline Height $(\mathrm{cm})$ & -0.536 & 0.246 & Height $(\mathrm{cm})$ & -0.606 & 0.182 \\
\hline No. of children & -1.160 & 0.384 & No of children & -1.399 & 0.289 \\
\hline Constant & 1708.474 & 0.001 & Constant & 1697.579 & 0.001 \\
\hline \multicolumn{3}{|l|}{ Final adjusted $R^{2}=0.238$} & \multicolumn{3}{|l|}{ Final adjusted $R^{2}=0.258$} \\
\hline
\end{tabular}

Subsequent studies of association between ER- $\alpha$ genotypes and BMD have showed mixed results. For instance, in postmenopausal Korean women, Han et al. (1997) failed to find any significant association between the ER- $\alpha$ genotypes and BMD in a hospital-based study, whereas Nam et al. (2005) observed the association of the $P v u$ II polymorphism of ER- $\alpha$ with BMD, and Kim et al. (2001) revealed the association of both $P v u \mathrm{II}$ and $X b a \mathrm{I}$ polymorphisms with BMD in population-based studies. These controversial results may be explained partly by an insufficient sample size, population specificity and possible genetic effects masked by different gene-gene and gene-environment interactions.

Because osteoporosis is known as a lifestyle-related disease, we took into account the analysis of both genetic factors and environmental factors, including: (1) anthropometric measurements: weight and height; (2) reproductive status: age, age at menopause, years since menopause and number of births; (3) lifestyle factors: educational level and occupation. As all subjects were healthy women who did not smoke and drink alcohol and most of them (98\%) were farmers and manual workers, we could eliminate the confounding factors of these variables in the all analysis models. It is the first report of the relationship between genetic factors, environmental factors and calcaneal SOS in Vietnam. To date, there have been few previous reports regarding to relationship between the ER- $\alpha P v u \mathrm{II}$ and $X b a \mathrm{I}$ genotypes and QUS parameters, although the QUS measurement has many advantages, such as noninvasive assessment of skeletal status, which is especially usefulness for pregnant women, low cost, portable tools and feasibility for a large population study. In agreement with the findings of Patel et al. (2000) and Koh et al. (2004), we found that the $\mathrm{x}$ allele of the ER- $\alpha$ gene was significantly associated with the reduced QUS parameter, while Albagha et al. (2005) observed no association. Interestingly, we also revealed the association of the $\mathrm{P}$ allele with increased QUS parameters, which is inconsistent with previous studies (Patel et al. 2000; Koh et al. 2004; Albagha et al. 2005). Our results can be explained by the hypothesis of Khosla et al. (2004) that the pp or xx genotype may be relatively estrogen insensitive and that subjects with the $\mathrm{P}$ or $\mathrm{X}$ allele may benefit more from the protective effects of estrogen on bone than subjects with the $p$ or $\mathrm{x}$ allele. Thus, positive or negative associations may be dependent on circulating of the estrogen level.

With regard to the relationship between the $P v u I I$, $X b a \mathrm{I}$ genotype of the ER- $\alpha$ gene and BMD in postmenopausal Asian populations, some studies reported that women with the XX genotype had higher BMDs than those with the $\mathrm{Xx}$ and/or xx genotype (Koh et al. 2004 and Mitra et al. 2006), while others found the opposite results (Kim et al. 2001 and Thuy et al. 2003). Similarly, the $\mathrm{P}$ allele was also associated with a higher BMD in most of the reports (Nam et al. 2005; Kim et al. 2001; Mitra et al. 2006), whereas only one reported an opposite association (Huang et al. 1999). The recent meta-analyses in Caucasian women, Ioannidis et al. (2004) confirmed that XX homozygotes consistently had a higher BMD and also a decreased risk of fractures when compared with carriers of the $\mathrm{x}$ allele, whereas the $P v u I I$ genotype was not associated with either BMD or fracture risk.

In terms of haplotype, we found that both px and PX haplotypes were associated with calcaneal SOS. Albagha et al. (2005), in the population-based cohort of 3,054 Scottish women, indicated that the px haplotype was associated with reduced calcaneal broadband ultrasound attenuation values. Surprisingly, our report is the first of an association between the PX haplotype and QUS parameter in postmenopausal women, while previous studies observed no association or did not carry out an analysis (Patel et al. 2000; Koh et al. 2004; Albagha et al. 2005). Other studies reported a haplotype analysis for the ER- $\alpha$ gene in relation to BMD, 
deriving inconsistent conclusions concerning the effects of the different haplotypes among different ethnic populations. Some studies indicated that the px haplotype was associated with lower BMD (Van Meurs et al. 2003; Zhang et al. 2003), while others (Huang et al. 1999) reported the association of the PX haplotype with lower BMD and others demonstrated the association of the Px haplotype with higher BMD (Kobayashi et al. 1996; Albagha et al. 2001). These largely inconsistent results may derive from the diverse genotype and haplotype distributions and the differential degree of linkage disequilibrium of different haplotypes with underlying unknown functional mutations among ethnic groups. In this study, the distribution of $P v u \mathrm{II}-X b a \mathrm{I}$ haplotypes differed from that in several ethnic backgrounds of Asian and Caucasian women (Patel et al. 2000; Koh et al. 2004; Albagha et al. 2005). On the other hand, the controversial results can be explained by gene-gene interaction. For instance, Zhang et al. (2003) revealed that neither of the single polymorphisms of the ER- $\alpha P v u \mathrm{II}$ or $X b a \mathrm{I}$ and none of the four haplotypes were associated with BMD. However, with AA (or A allele) at the vitamin D-receptor locus, PP carriers had lower hip BMD than the non-carriers.

Predictors for reduced bone density and an increased risk of osteoporosis are very important to prevent a population from fracture effectively. The present data show that the copy number of the px and PX haploypes and the years since menopause are significant predictors of both calcaneal SOS and osteoporosis. Other studies reported that either the Px haplotype (Albagha et al. 2001) or px haplotype (Ioannidis et al. 2004) were independent predictors of BMD and osteoporosis in different ethnic populations. Increased age is a significant predictor of low SOS. This association was reported in previous studies (Thuy et al. 2003; Hien et al. 2005) and was also confirmed in our data. Our study also agreed with previous research reporting that an increased educational level was associated with a reduced risk of osteoporosis in Vietnamese women (Hien et al. 2005). It may be elucidated by the report of Woo et al. (1999) that showed that a higher level of education was associated with a healthier diet and a better education might directly influence bone health through the positive effect of better health knowledge on individuals' lifestyles and behaviors.

The present findings must be interpreted in the context of a number of potential limitations. First, using SOS measurements is not known as a "gold standard" to diagnosis osteoporosis in the hospital, but rather BMD measured by dual-energy X-ray absorptiometry. However, many studies have supported the view of using QUS to screen for osteoporosis at the community level (Gluer 1997; Danielson et al. 1999; Frost et al. 2001; Hien et al. 2005). The previous studies also applied a $T$-score threshold of $\leq-1.8$ to define osteoporosis by using QUS (Frost et al. 2001 and Hien el al. 2005). Thus, in the context of this study in developing countries at the public health level, we could only apply QUS and use a $T$-score threshold of $\leq-1.8$ to identify subjects with osteoporosis. Second, calcium intake of the subjects was not used in these findings to evaluate a potential effect of this variable in our results. Next, our exclusion criteria, including the use of bisphosphonates within 6 months, were inappropriate because the treatment with these drugs in the prior 6 months might affect the bone measurements (Stock et al. 1997). Nevertheless, none of the recruited subjects had ever taken any bisphosphonates in their medical history. Thus, we could eliminate this confounding factor in the present study. Lastly, our sample size was relatively small and might provide inflated estimates compared to reality (Ioannidis et al. 2001). Although it was designed to eliminate the effects of confounding factors and to detect the statistically significant difference among genotypes and haplotypes of ER- $\alpha$,it did not have enough power to carry out further analysis by stratums and interactions between genetic and environmental factors. However, although it wassmall, this could be considered as a representative sample of postmenopausal women in rural Vietnam without other ethnic admixtures.

In conclusion, it is the first report to suggest that the $P v u \mathrm{II}$ and $X b a \mathrm{I}$ genotypes and haplotypes of the ER- $\alpha$ gene, age, years since menopause and educational level have associations with bone density, as assessed by calcaneal SOS, and are significant predictors of osteoporosis in postmenopausal Vietnamese women.

Acknowledgments We are grateful to Miss Yukiko Yoshida and Miss Isoko Nomura for their kindly help and support. We wish to thank Professor Toshio Matsumoto and Youichi Sato,Ph.D., for advice with regard to the HapMap and the effect of bisphosphonates.

\section{References}

Albagha OME, Mcguigan FEA, Reid DM et al (2001) Estrogen receptor $\alpha$ gene polymorphisms and bone mineral density: haplotype analysis in women from the United Kingdom. J Bone Miner Res 16:128-134

Albagha OM, Pettersson U, Stewart A et al (2005) Association of oestrogen receptor alpha gene polymorphisms with postmenopausal bone loss, bone mass, and quantitative ultrasound properties of bone. J Med Genet 42(3):240-246

Anthony D Woolf, Kristina Åkesson (2003) Preventing fractures in elderly people. BMJ 327:89-95 
Cheng S, Fan B, Wang L et al (1999) Factors affecting broadband ultrasound attenuation results of the calcaneus using a gel-coupled quantitative ultrasound scanning system. Osteoporos Int 10:495-504

Danielson ME, Cauley JA, Baker CE et al (1999) Familial resemblance of bone mineral density (BMD) and calcaneal ultrasound attenuation: the BMD in mothers and daughters study. J Bone Miner Res 14:102-110

Devoto M, Shimoya K, Caminis J et al (1998) First-stage autosomal genome screen in extended pedigrees suggests genes predisposing to low bone mineral density on chromosomes 1p, 2p and 4q. Eur J Hum Genet 6:151157

Frost ML, Blake GM, Fogelman I (1999) Contact quantitative ultrasound: an evaluation of precision, fracture discrimination, age-related bone loss and applicability of the WHO criteria. Osteoporos Int 10:441-449

Frost ML, Blake GM, Fogelman I (2001) Quantitative ultrasound and bone mineral density are equally strongly associated with risk factors for osteoporosis. J Bone Miner Res 16:406-416

Gennari L, Merlotti D, De Paola V et al (2005) Estrogen receptor gene polymorphisms and the genetics of osteoporosis. Am J Epidemiol 161:307-320

Giguere Y, Rousseau F (2000) The genetics of osteoporosis: 'complexities and difficulties.' Clin Genet 57:161-169

Gluer CC (1997) Quantitative ultrasound techniques for the assessment of osteoporosis: expert agreement on current status. The international quantitative ultrasound consensus group. J Bone Miner Res 12:1280-1288

Han KO, Moon IG, Kang YS et al (1997) Non-association of estrogen receptor genotypes with bone mineral density and estrogen responsiveness to hormone replacement therapy in Korean post-menopausal women. J Clin Endocrinol Metab 82:991-995

Hien Vu TT, Nguyen CK, Yamamoto S et al (2005) Determining the prevalence of osteoporosis and related factors using quantitative ultrasound in Vietnamese adult women. Am J Epidemiol 161(9):824-830

Howard GM, Nguyen TV, Harris M et al (1998) Genetic and environmental contributions to the association between quantitative ultrasound and bone mineral density measurements: a twin study. J Bone Miner Res 13:13181327

Huang Q, Wang Q, Zhang L et al (1999) Relationship between bone mineral density and polymorphism of the estrogen receptor gene in healthy postmenopausal women in China. Chin Med J 112:832-835

Huopio J, Kroger H, Honkanen R et al (2004) Calcaneal ultrasound predicts early postmenopausal fractures as well as axial BMD. A prospective study of 422 women. Osteoporos Int 15(3):190-195

Ioannidis JP, Ntzani EE, Trikalinos TA et al (2001) Replication validity of genetic association studies. Nat Genet 29:306-309

Ioannidis JP, Ralston SH, Bennett ST et al (2004) Differential genetic effects of ESR1 gene polymorphisms on osteoporosis outcomes. JAMA 292(17):2105-2114

Johnson ML, Gong G, Kimberling W et al (1997) Linkage of a gene causing high bone mass to human chromosome 11 (11q12-13). Am J Hum Genet 60:1326-1332

Khosla S, Riggs BL, Atkinson EJ et al (2004) Relationship of estrogen receptor genotypes to bone mineral density and to rates of bone loss in men. J Clin Endocrinol Metab 89:1808-1816
Kim JG, Lim KS, Kim EK et al (2001) Association of vitamin D receptor and estrogen receptor gene polymorphisms with bone mass in postmenopausal Korean women. Menopause 8:222-228

Kobayashi S, Inoue S, Hosoi T et al (1996) Association of bone mineral density with polymorphism of the estrogen receptor gene. J Bone Miner Res 11:306-311

Koh JM, Nam-Goong IS, Hong JS et al (2004) Oestrogen receptor alpha genotype, and interactions between vitamin $\mathrm{D}$ receptor and transforming growth factor-beta1 genotypes are associated with quantitative calcaneal ultrasound in postmenopausal women. Clin Endocrinol 60:232-340

Korach KS (1994) Insights from the study of animals lacking functional estrogen receptor. Science 266:1524-1527

Masi L, Becherini L, Colli E et al (1998) Polymorphisms of the calcitonin receptor gene are associated with bone mineral density in postmenopausal Italian women. Biochem Biophys Res Commun 248:190-195

Mitra S, Desai M, Khatkhatay MI (2006) Association of estrogen receptor alpha gene polymorphisms with bone mineral density in postmenopausal Indian women. Mol Genet Metab 87(1):80-87

Morrison NA, Qi JC, Tokita A et al (1994) Prediction of bone density from vitamin D receptor alleles. Nature 367:284-287

Nam HS, Shin MH, Kweon SS et al (2005) Association of estrogen receptor-alpha gene polymorphisms with bone mineral density in postmenopausal Korean women. J Bone Miner Metab 23(1):84-89

Nordström P, Lorentzon R (1999) Influence of heredity and environment on bone density in adolescent boys: a parentoffspring study. Osteoporos Int 10:271-277

Patel MS, Cole DE, Smith JD et al (2000) Alleles of the estrogen receptor alpha-gene and an estrogen receptor cotranscriptional activator gene, amplified in breast cancer-1 (AIB1), are associated with quantitative calcaneal ultrasound. J Bone Miner Res 15(11):2231-2239

Smith EP, Boyd J, Frank GR et al (1994) Estrogen resistance caused by a mutation in the estrogen-receptor gene in a man. N Engl J Med 331:1056-1061

Spencer H, Kramer L (1986) NIH consensus conference: Osteoporosis, factors contributing to osteoporosis. J Nutr 116:319-322

Stock JL, Bell NH, Chesnut CH et al (1997) Increments in bone mineral density of the lumbar spine and hip and suppression of bone turnover are maintained after discontinuation of alendronate in postmenopausal women. Am J Med 103(4):291-297

Thuy VT, Chau TT, Nguyen TV et al (2003) Assessment of low bone mass in Vietnamese: comparison of QUS calcaneal ultrasonometer and data-derived T-scores. J Bone Miner Metab 21(2):114-119

Van Meurs JB, Schuit SC, Weel AE et al (2003) Association of 5' estrogen receptor alpha gene polymorphisms with bone mineral density vertebral bone area and fracture risk. Hum Mol Genet 12(14):1745-1754

Woo J, Leung SS, Ho SC et al (1999) Influence of educational level and marital status on dietary intake, obesity and other cardiovascular risk factors in a Hong Kong Chinese population. Eur J Clin Nutr 53:461-467

Zhang YY, Long JR, Liu PY et al (2003) Estrogen receptor $\alpha$ and vitamin $\mathrm{D}$ receptor gene polymorphisms and bone mineral density: association study of healthy pre- and postmenopausal Chinses women. Biochem Biophys Res Commun 308:777-783 